Research Article

\title{
On Caristi Type Maps and Generalized Distances with Applications
}

\author{
Wei-Shih Du \\ Department of Mathematics, National Kaohsiung Normal University, Kaohsiung 824, Taiwan \\ Correspondence should be addressed to Wei-Shih Du; wsdu@nknucc.nknu.edu.tw
}

Received 3 July 2013; Accepted 5 August 2013

Academic Editor: E. Karapinar

Copyright (C) 2013 Wei-Shih Du. This is an open access article distributed under the Creative Commons Attribution License, which permits unrestricted use, distribution, and reproduction in any medium, provided the original work is properly cited.

We prove some new existence theorems of fixed points for Caristi type maps and some suitable generalized distances without lower semicontinuity assumptions on dominated functions. As applications of our results, some new fixed point theorems and new generalizations of the Banach contraction principle are given.

\section{Introduction}

In 1972, Caristi proved the following famous fixed point theorem.

Theorem 1 (Caristi [1]). Let $(X, d)$ be a complete metric space and $f: X \rightarrow \mathbb{R}$ a lower semicontinuous and bounded below function. Suppose that $T$ is a Caristi type map on $X$ dominated by $f$; that is, $T$ satisfies

$$
d(x, T x) \leq f(x)-f(T x) \quad \text { for each } x \in X .
$$

Then $T$ has a fixed point in $X$.

It is well-known that the Caristi's fixed point theorem is one of the most valuable generalization of the Banach contraction principle [2], and it is equivalent to the Ekeland's variational principle, to the Takahashi's nonconvex minimization theorem, to the Daneš' drop theorem, to the petal theorem, and to the Oettli-Théra's theorem; see [3-26] and references therein for more details. A number of generalizations in various different directions of the Caristi's fixed point theorem have been investigated by several authors; see, for example, [4-30] and references therein. An interesting direction of research is the extension of Caristi's fixed point theorem, Ekeland's variational principle, and Takahashi's nonconvex minimization theorem to generalized distances, for example, $w$-distances $[5,10,14,19], \tau$-distances [11, 12 , $22], \tau$-functions $[13,15,18,22-25,31-36]$, weak $\tau$-functions
[24, 25], $P$-distances [26], $Q$-functions [21], generalized pseudodistances $[22,23]$, and others. For more details on these generalizations, one can refer to $[5,10-26]$ and references therein.

Let us recall how we can exploit Caristi's fixed point theorem to prove the Banach contraction principle. A selfmap $T$ on a metric space $(X, d)$ is called contractive or Banach type if there exists a real number $\lambda \in[0,1)$ such that

$$
d(T x, T y) \leq \lambda d(x, y) \quad \text { for any } x, y \in X .
$$

It is obvious that if $T$ is a contractive map on $X$, then $T$ is continuous on $X$ and (2) will deduce the following inequality:

$$
\begin{array}{r}
d(x, T x) \leq \frac{1}{1-\lambda} d(x, T x)-\frac{1}{1-\lambda} d\left(T x, T^{2} x\right) \\
\text { for any } x \in X .
\end{array}
$$

The inequality (3) admits that $T$ is a Caristi type map on $X$ dominated by $f$ defined by $f(x):=(1 /(1-\lambda)) d(x, T x)$. From the continuity of $T$, the function $f$ is continuous on $X$, and therefore the Caristi's fixed point theorem is applicable to prove the Banach contraction principle. It is quite obvious that for any map $T$ and any generalized distances $p$, the function $x \mapsto p(x, T x)$ is not necessarily to be continuous even lower semicontinuous, so such well-known generalized versions of Caristi's fixed point theorem with lower semicontinuity are not easily applicable to any generalized version 
of Banach contraction principle for generalized distances. Motivated by the reason, in the recently paper [20], the author established some new versions of Caristi type fixed point theorem such that they can be applicable to prove generalized versions of Banach contraction principle for suitable generalized distances.

This work can be considered as a continuation of the paper [20]. In this paper, we first establish some new fixed point theorems for Caristi type maps and some suitable generalized distances without assuming that the dominated functions possess lower semicontinuity property. As applications of our results, some new fixed point theorems and new generalizations of the Banach contraction principle are given. We have already succeeded in utilizing our new versions of Caristi type fixed point theorem to deal with the existence results for any map $T$ satisfying

$$
d(T x, T y) \leq \alpha(d(x, y)) d(x, y) \quad \forall x, y \in X,
$$

where $\alpha:[0,+\infty) \rightarrow[0,1)$ is a function satisfying $\limsup _{s \rightarrow t^{+}} \alpha(s)<1$ for all $t \in[0,+\infty)$.

\section{Preliminaries}

We recall in this section the notations, definitions, and results needed. Let $(X, d)$ be a metric space. An extended real valued function $\phi: X \rightarrow(-\infty,+\infty)$ is said to be lower semicontinuous (l.s.c., for short) at $w \in X$ if for any sequence $\left\{x_{n}\right\}$ in $X$ with $x_{n} \rightarrow w$ as $n \rightarrow \infty$, we have $\phi(w) \leq$ $\liminf _{n \rightarrow \infty} \phi\left(x_{n}\right)$. The function $\phi$ is called to be l.s.c. on $X$ if $\phi$ is l.s.c. at every point of $X$. The function $\phi$ is said to be proper if $\phi \not \equiv+\infty$. Let $T: X \rightarrow X$ be a selfmap. $T$ is said to be closed if $G r T=\{(x, y) \in X \times X: y=T x\}$, the graph of $T$, is closed in $X \times X$. A point $v$ in $X$ is a fixed point of $T$ if $T v=v$. The set of fixed points of $T$ is denoted by $\mathscr{F}(T)$. Throughout this paper we denote by $\mathbb{N}$ and $\mathbb{R}$, the set of positive integers and nonnegative real numbers, respectively.

Recall that a function $p: X \times X \rightarrow[0,+\infty)$ is called a $w$ distance [5, 10-20, 31], first introduced and defined by Kada, Suzuki, and Takahashi, if the following are satisfied:

(w1) $p(x, z) \leq p(x, y)+p(y, z)$ for any $x, y, z \in X$;

$(w 2)$ for any $x \in X, p(x, \cdot): X \rightarrow[0,+\infty)$ is l.s.c.;

(w3) for any $\varepsilon>0$, there exists $\delta>0$ such that $p(z, x) \leq \delta$ and $p(z, y) \leq \delta$ imply $d(x, y) \leq \varepsilon$.

A function $p: X \times X \rightarrow[0,+\infty)$ is said to be a $\tau$-function $[13,15,18,22-25,31-36]$, first introduced and studied by Lin and $\mathrm{Du}$, if the following conditions hold:

( $\tau 1) p(x, z) \leq p(x, y)+p(y, z)$ for all $x, y, z \in X$;

( $\tau 2)$ if $x \in X$ and $\left\{y_{n}\right\}$ in $X$ with $\lim _{n \rightarrow \infty} y_{n}=y$ such that $p\left(x, y_{n}\right) \leq M$ for some $M=M(x)>0$, then $p(x, y) \leq$ $M$;

( $\tau 3)$ for any sequence $\left\{x_{n}\right\}$ in $X$ with $\lim _{n \rightarrow \infty} \sup \left\{p\left(x_{n}\right.\right.$, $\left.\left.x_{m}\right): m>n\right\}=0$, if there exists a sequence $\left\{y_{n}\right\}$ in $X$ such that $\lim _{n \rightarrow \infty} p\left(x_{n}, y_{n}\right)=0$, then $\lim _{n \rightarrow \infty} d\left(x_{n}\right.$, $\left.y_{n}\right)=0$;
( $\tau 4)$ for $x, y, z \in X, p(x, y)=0$ and $p(x, z)=0$ imply $y=z$.

Note that not either of the implications $p(x, y)=0 \Leftrightarrow$ $x=y$ necessarily holds and $p$ is nonsymmetric in general. It is well known that the metric $d$ is a $w$-distance and any $w$ distance is a $\tau$-function, but the converse is not true; see [13, 31] for more detail.

Example 2 (see [31, Example A]). Let $X=\mathbb{R}$ with the metric $d(x, y)=|x-y|$ for $x, y \in X$, and $0<a<b$. Define the function $p: X \times X \rightarrow[0,+\infty)$ by

$$
p(x, y)=\max \{a(y-x), b(x-y)\} \text {. }
$$

Then $p$ is a $\tau$-function.

The following result is crucial in this paper.

Theorem 3 (see [18, Lemma 2.1]). Let $(X, d)$ be a metric space and $p: X \times X \rightarrow[0,+\infty)$ a function. Assume that $p$ satisfies the condition ( $\tau 3)$. If a sequence $\left\{x_{n}\right\}$ in $X$ with $\lim _{n \rightarrow \infty} \sup \left\{p\left(x_{n}, x_{m}\right): m>n\right\}=0$, then $\left\{x_{n}\right\}$ is a Cauchy sequence in $X$.

Recently, the concepts of weak $\tau$-function and generalized pseudodistance were introduced and studied by Khanh and Quy [24, 25] and Włodarczyk and Plebaniak [22] as follows.

Definition 4. Let $(X, d)$ be a metric space. A function $p: X \times$ $X \rightarrow[0,+\infty)$ is called

(i) a weak $\tau$-function $[24,25]$ on $X$ if conditions $(\tau 1)$, $(\tau 3)$, and ( $\tau 4)$ hold;

(ii) a generalized pseudodistance [22] on $X$ if conditions $(\tau 1)$ and $(\tau 3)$ hold.

It is obvious that any $\tau$-function is a weak $\tau$-function and every weak $\tau$-function is a generalized pseudodistance, but the converse parts are not always true. The first observation is that there exists a weak $\tau$-function which is not a $\tau$-function.

Example 5 (see [24, Example 2.5]). Let $X=[0,+\infty), \gamma>0$, and $p: X \times X \rightarrow[0,+\infty)$ be defined by

$$
p(x, y)= \begin{cases}|x-y|+\gamma, & \text { if } x \neq y, \\ \frac{3}{2} \gamma, & \text { if } x=y .\end{cases}
$$

Then $p$ is a weak $\tau$-function which is neither a $\tau$-function nor a $w$-distance.

The following example shows that there exists a generalized pseudodistance which is not a weak $\tau$-function.

Example 6 (see [22, Example 1.3]). Define a function $p$ : $[0,2] \times[0,2] \rightarrow[0,+\infty)$ by

$$
p(x, y)= \begin{cases}0, & \text { if } x-y=-2 \\ |x-y|, & \text { if }-2<x-y \leq 0 \\ x-y+2, & \text { if } 0<x-y \leq 2\end{cases}
$$


Then $p$ is a generalized pseudodistance but not a weak $\tau$ function.

Very recently, the author first introduced the following concepts.

Definition 7 (see [20]). Let $(X, d)$ be a metric space, and let $f: X \rightarrow \mathbb{R}, \varphi: \mathbb{R} \rightarrow(0,+\infty)$, and $p: X \times X \rightarrow[0,+\infty)$ be functions. A single-valued selfmap $T: X \rightarrow X$ is called

(i) Caristi type on $X$ dominated by $p, \varphi$, and $f$ (abbreviated as $(p, \varphi, f)$-Caristi type on $X)$ if

$p(x, T x) \leq \varphi(f(x))(f(x)-f(T x)) \quad$ for each $x \in X$

(ii) Caristi type on $X$ dominated by $p$ and $f$ (abbreviated as $(p, f)$-Caristi type on $X)$ if

$$
p(x, T x) \leq f(x)-f(T x) \quad \text { for each } x \in X ;
$$

(iii) Caristi type on $X$ dominated by $\varphi$ and $f$ (abbreviated as $(\varphi, f)$-Caristi type on $X)$ if

$d(x, T x) \leq \varphi(f(x))(f(x)-f(T x)) \quad$ for each $x \in X$

(iv) Caristi type on $X$ dominated by $f$ (abbreviated as $(f)$ Caristi type on $X$ ) if

$d(x, T x) \leq f(x)-f(T x) \quad$ for each $x \in X$.

Clearly, if $T$ is $(p, f)$-Caristi type (resp. $(f)$-Caristi type) on $X$, then $T$ is $(p, \varphi, f)$-Caristi type (resp. $(\varphi, f)$-Caristi type) on $X$ with $\varphi(t)=1$ for all $t$. The following example illustrates that their converse are not always true.

Example 8. Let $X=[0,+\infty)$ with the usual metric $d(x, y)=$ $|x-y|$. Then $(X, d)$ is a complete metric space. Let $p: X \times$ $X \rightarrow[0,+\infty)$ be defined by

$$
p(x, y)=\max \{20(x-y), 40(y-x)\},
$$

for all $x, y \in X$. By Example 2, we know that $p$ is a $\tau$-function. Let $T: X \rightarrow X$ be defined by $T x=x^{2}, x \in X$. Define $f: X \rightarrow \mathbb{R}$ by

$$
f(x)= \begin{cases}4 x-12, & \text { if } x \in[0,1) \\ 15-8 x, & \text { if } x \in[1,+\infty) .\end{cases}
$$

Then $f$ is not lower semicontinuous at $x=1$. For $i=1,2$, let $\varphi_{i}: \mathbb{R} \rightarrow(0,+\infty)$ be defined by

$$
\varphi_{1}(t)=2, \quad \varphi_{2}(t)=6 \quad \forall t \in \mathbb{R},
$$

respectively. For $x \in[0,1)$, we have

$$
\begin{gathered}
d(x, T x)=x-x^{2}<4\left(x-x^{2}\right)=f(x)-f(T x) \\
p(x, T x)=\max \{20(x-T x), 40(T x-x)\} \\
=20\left(x-x^{2}\right) \\
<\varphi_{2}(f(x))(f(x)-f(T x)) .
\end{gathered}
$$

For $x \in[1,+\infty)$, we have

$$
\begin{gathered}
d(x, T x)=x^{2}-x<8\left(x^{2}-x\right)=f(x)-f(T x), \\
p(x, T x)=40\left(x^{2}-x\right)<\varphi_{2}(f(x))(f(x)-f(T x)) .
\end{gathered}
$$

Hence, for any $x \in X$, we show

$$
\begin{aligned}
d(x, T x) & \leq f(x)-f(T x) \\
& \leq \varphi_{i}(f(x))(f(x)-f(T x)) \text { for each } i \in\{1,2\}, \\
& p(x, T x) \leq \varphi_{2}(f(x))(f(x)-f(T x)) .
\end{aligned}
$$

So, $T$ is $(f)$-Caristi type on $X$ as well as $\left(\varphi_{i}, f\right)$-Caristi type on $X$ for all $i \in\{1,2\}$. Moreover, we know that $T$ is $\left(p, \varphi_{2}, f\right)$ Caristi type on $X$, but it is neither $(p, f)$-Caristi type nor $\left(p, \varphi_{1}, f\right)$-Caristi type on $X$ based on the following fact

$$
p(x, T x)>\varphi_{1}(f(x))(f(x)-f(T x))>f(x)-f(T x)
$$

$\forall x \in X$.

Definition 9 (see [31-36]). A function $\alpha:[0,+\infty) \rightarrow[0,1)$ is said to be an $\mathscr{M} \mathscr{T}$-function (or $\mathscr{R}$-function) if lim $\sup _{s \rightarrow t^{+}}$ $\alpha(s)<1$ for all $t \in[0,+\infty)$.

It is obvious that if $\alpha:[0,+\infty) \rightarrow[0,1)$ is a nondecreasing function or a nonincreasing function, then $\alpha$ is an $\mathscr{M} \mathscr{T}$-function. So the set of $\mathscr{M} \mathscr{T}$-functions is a rich class. But it is worth to mention that there exist functions which are not $\mathscr{M}$-functions.

Example 10 (see [32]). Let $\alpha:[0,+\infty) \rightarrow[0,1)$ be defined by

$$
\alpha(t):= \begin{cases}\frac{\sin t}{t}, & \text { if } t \in\left(0, \frac{\pi}{2}\right] \\ 0, & \text { otherwise. }\end{cases}
$$

Since $\lim \sup _{s \rightarrow 0^{+}} \alpha(s)=1, \varphi$ is not an $\mathscr{M} \mathscr{T}$-function.

Recently, Du [32] first proved the following characterizations of $\mathscr{M} \mathscr{T}$-functions.

Theorem 11 (see [32]). Let $\alpha:[0,+\infty) \rightarrow[0,1)$ be a function. Then the following statements are equivalent.

(a) $\alpha$ is an $\mathscr{M} \mathscr{T}$-function.

(b) For each $t \in[0,+\infty)$, there exist $r_{t}^{(1)} \in[0,1)$ and $\varepsilon_{t}^{(1)}>$ 0 such that $\alpha(s) \leq r_{t}^{(1)}$ for all $s \in\left(t, t+\varepsilon_{t}^{(1)}\right)$.

(c) For each $t \in[0,+\infty)$, there exist $r_{t}^{(2)} \in[0,1)$ and $\varepsilon_{t}^{(2)}>$ 0 such that $\alpha(s) \leq r_{t}^{(2)}$ for all $s \in\left[t, t+\varepsilon_{t}^{(2)}\right]$.

(d) For each $t \in[0,+\infty)$, there exist $r_{t}^{(3)} \in[0,1)$ and $\varepsilon_{t}^{(3)}>$ 0 such that $\alpha(s) \leq r_{t}^{(3)}$ for all $s \in\left(t, t+\varepsilon_{t}^{(3)}\right]$.

(e) For each $t \in[0,+\infty)$, there exist $r_{t}^{(4)} \in[0,1)$ and $\varepsilon_{t}^{(4)}>$ 0 such that $\alpha(s) \leq r_{t}^{(4)}$ for all $s \in\left[t, t+\varepsilon_{t}^{(4)}\right)$. 
(f) For any nonincreasing sequence $\left\{x_{n}\right\}_{n \in \mathbb{N}}$ in $[0,+\infty)$, one has $0 \leq \sup _{n \in \mathbb{N}} \alpha\left(x_{n}\right)<1$.

(g) $\alpha$ is a function of contractive factor; that is, for any strictly decreasing sequence $\left\{x_{n}\right\}_{n \in \mathbb{N}}$ in $[0,+\infty)$, one has $0 \leq \sup _{n \in \mathbb{N}} \alpha\left(x_{n}\right)<1$.

\section{New Results for Caristi Type Maps and Their Applications}

We start with the following useful auxiliary result.

Theorem 12. Let $(X, d)$ be a metric space, $f: X \rightarrow(-\infty$, $+\infty$ ] a proper and bounded below function, $\varphi: \mathbb{R} \rightarrow(0,+\infty)$ a nondecreasing function, $p: X \times X \rightarrow[0, \infty)$ a function, and $T: X \rightarrow X$ a selfmap on $X$. Let $u \in X$ with $f(u)<+\infty$. Define $x_{1}=u$ and $x_{n+1}=T x_{n}$ for each $n \in \mathbb{N}$. If $p$ satisfies $(\tau 1)$ and $T$ is $(p, \varphi, f)$-Caristi type on $X$, then

$$
\lim _{n \rightarrow \infty} \sup \left\{p\left(x_{n}, x_{m}\right): m>n\right\}=0 .
$$

Moreover, if we futher assume that $p$ satisfies $(\tau 3)$, then $\left\{x_{n}\right\}_{n \in \mathbb{N}}$ is a Cauchy sequence in $X$.

Proof. For $x_{1}=u, f\left(x_{1}\right)<+\infty$. Since $T$ is $(p, \varphi, f)$-Caristi type on $X$, we get

$$
\begin{aligned}
p\left(x_{1}, x_{2}\right) & =p\left(x_{1}, T x_{1}\right) \\
& \leq \varphi\left(f\left(x_{1}\right)\right)\left(f\left(x_{1}\right)-f\left(T x_{1}\right)\right) \\
& =\varphi\left(f\left(x_{1}\right)\right)\left(f\left(x_{1}\right)-f\left(x_{2}\right)\right),
\end{aligned}
$$

which implies

$$
f\left(x_{2}\right) \leq f\left(x_{1}\right)<+\infty .
$$

Similarly, we have

$$
\begin{gathered}
p\left(x_{2}, x_{3}\right)=p\left(x_{2}, T x_{2}\right) \leq \varphi\left(f\left(x_{2}\right)\right)\left(f\left(x_{2}\right)-f\left(x_{3}\right)\right), \\
f\left(x_{3}\right) \leq f\left(x_{2}\right) \leq f\left(x_{1}\right)<+\infty .
\end{gathered}
$$

Hence, by induction, we can obtain the following inequalities

$$
\begin{aligned}
p\left(x_{n}, x_{n+1}\right) & \leq \varphi\left(f\left(x_{n}\right)\right)\left(f\left(x_{n}\right)-f\left(x_{n+1}\right)\right), \\
f\left(x_{n+1}\right) & \leq f\left(x_{n}\right)<+\infty \quad \text { for each } n \in \mathbb{N} .
\end{aligned}
$$

Since $f$ is bounded below,

$$
r:=\lim _{n \rightarrow \infty} f\left(x_{n}\right)=\inf _{n \in \mathbb{N}} f\left(x_{n}\right) \text { exists. }
$$

By (25), since $\varphi$ is nondecreasing, we have

$$
\varphi\left(f\left(x_{n}\right)\right) \leq \varphi\left(f\left(x_{1}\right)\right) \quad \forall n \in \mathbb{N} .
$$

For $m>n$ with $m, n \in \mathbb{N}$, taking into account $(\tau 1),(24),(26)$, and (27), we get

$$
p\left(x_{n}, x_{m}\right) \leq \sum_{j=n}^{m-1} p\left(x_{j}, x_{j+1}\right) \leq \varphi\left(f\left(x_{1}\right)\right)\left(f\left(x_{n}\right)-r\right) .
$$

Let $\alpha_{n}=\varphi\left(f\left(x_{1}\right)\right)\left(f\left(x_{n}\right)-r\right), n \in \mathbb{N}$. Then $\sup \left\{p\left(x_{n}, x_{m}\right)\right.$ : $m>n\} \leq \alpha_{n}$ for each $n \in \mathbb{N}$. Since $\lim _{n \rightarrow \infty} f\left(x_{n}\right)=r$, we obtain $\lim _{n \rightarrow \infty} \alpha_{n}=0$, and hence $\lim _{n \rightarrow \infty} \sup \left\{p\left(x_{n}, x_{m}\right)\right.$ : $m>n\}=0$. Moreover, if $p$ satisfies $(\tau 3)$, then the desired conclusion follows from Theorem 3 immediately. The proof is completed.

Applying Theorem 12, we prove a new fixed point theorem for Caristi type maps and generalized pseudodistances. It is worth to mention that in Theorem 13 we pose some suitable assumptions on the map $T$ without assuming that the domi-nated functions possess lower semicontinuity property.

Theorem 13. Let $(X, d)$ be a complete metric space, $f$ : $X \rightarrow(-\infty,+\infty]$ a proper and bounded below function, $\varphi$ : $\mathbb{R} \rightarrow(0,+\infty)$ a nondecreasing function, and $p$ a generalized pseudodistance on $X$. Suppose that $T: X \rightarrow X$ is a $(p, \varphi, f)$ Caristi type selfmap on $X$ and one of the following conditions is satisfied:

(H1) $T$ is continuous;

(H2) $T$ is closed;

(H3) $p(x, y)=0$ implies $x=y$ for all $x, y \in X$ and the map $g: X \rightarrow[0, \infty)$ defined by $g(x)=p(x, T x)$ is l.s.c.;

$(\mathrm{H} 4)$ the map $h: X \rightarrow[0, \infty)$ defined by $h(x)=d(x, T x)$ is l.s.c.;

(H5) for any sequence $\left\{z_{n}\right\}$ in $X$ with $z_{n+1}=T z_{n}, n \in \mathbb{N}$ and $\lim _{n \rightarrow \infty} z_{n}=a$, we have $\lim _{n \rightarrow \infty} p\left(z_{n}, T a\right)=0$.

Then $T$ admits a fixed point in $X$. Moreover, for any $w \in X$ with $f(w)<+\infty$, the sequence $\left\{T^{n} w\right\}_{n \in \mathbb{N}}$ converges to a fixed point of $T$.

Proof. Let $S=\{x \in X: f(x)<+\infty\}$. Since $f$ is proper, $S \neq \emptyset$. Let $w \in S$. Define $x_{1}=w$ and $x_{n+1}=T x_{n}=T^{n} w$ for each $n \in \mathbb{N}$. Since $p$ is a generalized pseudodistance on $X$, by applying Theorem 12 , we know that $\left\{x_{n}\right\}_{n \in \mathbb{N}}$ is a Cauchy sequence in $X$ and

$$
\lim _{n \rightarrow \infty} \sup \left\{p\left(x_{n}, x_{m}\right): m>n\right\}=0
$$

The last equality implies

$$
\lim _{n \rightarrow \infty} p\left(x_{n}, x_{n+1}\right)=0
$$

By the completeness of $X$, there exists $v_{w} \in X$ such that $x_{n} \rightarrow$ $v_{w}$ as $n \rightarrow \infty$.

Now, we verify $v_{w} \in \mathscr{F}(T)$. If (H1) holds, since $T$ is continuous on $X, x_{n+1}=T x_{n}$ for each $n \in \mathbb{N}$ and $x_{n} \rightarrow v_{w}$ as $n \rightarrow$ $\infty$, we get

$$
v_{w}=\lim _{n \rightarrow \infty} x_{n}=\lim _{n \rightarrow \infty} x_{n+1}=\lim _{n \rightarrow \infty} T x_{n}=T\left(\lim _{n \rightarrow \infty} x_{n}\right)=T v_{w},
$$

which means $v_{w} \in \mathscr{F}(T)$. If (H2) holds, since $T$ is closed, $x_{n+1}=T x_{n}$ for each $n \in \mathbb{N}$ and $x_{n} \rightarrow v_{w}$ as $n \rightarrow \infty$, we have $T v_{w}=v_{w}$. Suppose that $(\mathrm{H} 3)$ holds. By the lower 
semicontinuity of $g, x_{n} \rightarrow v_{w}$ as $n \rightarrow \infty$ and (30), we obtain

$$
\begin{aligned}
p\left(v_{w}, T v_{w}\right) & =g\left(v_{w}\right) \leq \liminf _{n \rightarrow \infty} g\left(x_{n}\right) \\
& =\lim _{n \rightarrow \infty} p\left(x_{n}, x_{n+1}\right)=0,
\end{aligned}
$$

which implies $p\left(v_{w}, T v_{w}\right)=0$. By the hypothesis in (H3), we get $v_{w} \in \mathscr{F}(T)$. Suppose that (H4) holds. Since $\left\{x_{n}\right\}_{n \in \mathbb{N}}$ is convergent in $X$,

$$
\lim _{n \rightarrow \infty} d\left(x_{n}, x_{n+1}\right)=0 .
$$

Since

$$
d\left(v_{w}, T v_{w}\right)=h\left(v_{w}\right) \leq \liminf _{n \rightarrow \infty} d\left(x_{n}, x_{n+1}\right)=0,
$$

we obtain $d\left(v_{w}, T v_{w}\right)=0$, and hence $v_{w} \in \mathscr{F}(T)$. Finally, assume (H5) holds. Since $\lim _{n \rightarrow \infty} \sup \left\{p\left(x_{n}, x_{m}\right): m>n\right\}=$ 0 and $\lim _{n \rightarrow \infty} p\left(x_{n}, T v_{w}\right)=0$, there exists $\left\{a_{n}\right\} \subset\left\{x_{n}\right\}$ with $\lim _{n \rightarrow \infty} \sup \left\{p\left(a_{n}, a_{m}\right): m>n\right\}=0$ and $b_{n}=T v_{w}$ for all $n \in \mathbb{N}$, such that $\lim _{n \rightarrow \infty} p\left(a_{n}, b_{n}\right)=0$. By $(\tau 3)$, $\lim _{n \rightarrow \infty} d\left(a_{n}, b_{n}\right)=0$. Since $a_{n} \rightarrow v_{w}$ as $n \rightarrow \infty$ and $d\left(b_{n}, v_{w}\right) \leq d\left(b_{n}, a_{n}\right)+d\left(a_{n}, v_{w}\right)$, we get $b_{n} \rightarrow v_{w}$ as $n \rightarrow \infty$. So $T v_{w}=v_{w}$ or $v_{w} \in \mathscr{F}(T)$. Therefore, in any case, we prove $v_{w} \in \mathscr{F}(T)$. Since $w \in S$ is arbitrary, the sequence $\left\{T^{n} w\right\}_{n \in \mathbb{N}}$ converges to a fixed point $v_{w}$ of $T$. This completes the proof.

Here, we give an example illustrating Theorem 13. This example also gives a negative answer to the uniqueness of fixed point.

Example 14. Let $X=[0,1]$ with the usual metric $d(x, y)=$ $|x-y|$. Then $(X, d)$ is a complete metric space. Define $p$ : $X \times X \rightarrow[0,+\infty)$ by

$$
p(x, y)=\max \{2(x-y), 3(y-x)\},
$$

for all $x, y \in X$. Then $p$ is a generalized pseudodistance on $X$. Let $f: X \rightarrow \mathbb{R}$ and $\varphi: \mathbb{R} \rightarrow(0,+\infty)$ be defined by

$$
\begin{gathered}
f(x)= \begin{cases}\frac{1}{3} x-25, & \text { if } x \in\left[0, \frac{1}{2}\right) \\
\frac{1}{2} x-3, & \text { if } x \in\left[\frac{1}{2}, 1\right],\end{cases} \\
\varphi(t)=10 \quad \forall t \in \mathbb{R},
\end{gathered}
$$

respectively. So $f(x)<+\infty$ for all $x \in X$. Note that $f$ is not lower semicontinuous at $x=1 / 2$, so $f$ is not lower semicontinuous on $X$. Since $f(x) \geq-25$ for all $x \in X, f$ is a bounded below function on $X$. Let $T: X \rightarrow X$ be defined by

$$
T x=x^{2} \quad \forall x \in X
$$

Then $T$ is continuous on $X$ and $\mathscr{F}(T)=\{0,1\}$. It is also easy to see that $T$ is closed and the map $x \mapsto d(x, T x)$ is l.s.c. Hence
(H1), (H2), and (H4) as in Theorem 13 hold. We deduce that for any $x \in X$,

$$
\begin{aligned}
p(x, T x) & =\max \{2(x-T x), 3(T x-x)\} \\
& =2\left(x-x^{2}\right) \\
& <\varphi(f(x))(f(x)-f(T x)),
\end{aligned}
$$

so $T$ is $(p, \varphi, f)$-Caristi type on $X$. Let $w \in X$. Since

$$
\lim _{n \rightarrow \infty} T^{n} w=\lim _{n \rightarrow \infty} w^{2 n}= \begin{cases}0, & \text { if } w \in[0,1) \\ 1, & \text { if } w=1,\end{cases}
$$

we know that $\left\{T^{n} w\right\}_{n \in \mathbb{N}}$ converges and the limit of $\left\{T^{n} w\right\}_{n \in \mathbb{N}}$ belongs to $\mathscr{F}(T)=\{0,1\}$. On the other hand, since all the assumptions of Theorem 13 are satisfied, by applying Theorem 13, we also prove that $T$ has a fixed point in $X$ and for any $w \in X$, the sequence $\left\{T^{n} w\right\}_{n \in \mathbb{N}}$ converges to a fixed point of $T$. It is worth noticing that any well-known generalized version of Caristi's fixed point theorem is not applicable here.

The following conclusions are immediate from Theorem 13 .

Corollary 15. Let $(X, d)$ be a complete metric space, $f: X \rightarrow$ $(-\infty,+\infty]$ a proper and bounded below function, and $p$ a generalized pseudodistance on $X$. Suppose that $T: X \rightarrow X$ is a $(p, f)$-Caristi type selfmap on $X$ and one of the conditions (H1), (H2), (H3), (H4), and (H5) as in Theorem 13 holds. Then $T$ admits a fixed point in $X$. Moreover, for any $w \in X$ with $f(w)<+\infty$, the sequence $\left\{T^{n} w\right\}_{n \in \mathbb{N}}$ converges to a fixed point of $T$.

Corollary 16. Let $(X, d)$ be a complete metric space, $f: X \rightarrow$ $(-\infty,+\infty]$ a proper and bounded below function, and $\varphi$ : $\mathbb{R} \rightarrow(0,+\infty)$ a nondecreasing function. Suppose that $T:$ $X \rightarrow X$ is a $(\varphi, f)$-Caristi type selfmap on $X$ and one of the following conditions is satisfied:

(D1) $T$ is continuous;

(D2) $T$ is closed;

(D3) the map $h: X \rightarrow[0, \infty)$ defined by $h(x)=d(x, T x)$ is l.s.c.

Then $T$ admits a fixed point in $X$. Moreover, for any $w \in X$ with $f(w)<+\infty$, the sequence $\left\{T^{n} w\right\}_{n \in \mathbb{N}}$ converges to a fixed point of $T$.

Corollary 17. Let $(X, d)$ be a complete metric space and $f$ : $X \rightarrow(-\infty,+\infty]$ a proper and bounded below function. Suppose that $T: X \rightarrow X$ is a $(f)$-Caristi type selfmap on $X$ and one of the conditions (D1), (D2), and (D3) as in Corollary 16 holds. Then $T$ admits a fixed point in X. Moreover, for any $w \in X$ with $f(w)<+\infty$, the sequence $\left\{T^{n} w\right\}_{n \in \mathbb{N}}$ converges to a fixed point of $T$.

Now, we give another quite useful auxiliary theorem for our applications. 
Theorem 18. Let $(X, d)$ be a metric space, $p: X \times X \rightarrow$ $[0,+\infty)$ a function, and $T: X \rightarrow X$ a selfmap. Suppose that there exists an $\mathscr{M} \mathscr{T}$-function $\alpha:[0,+\infty) \rightarrow[0,1)$ such that

$$
p(T x, T y) \leq \alpha(p(x, y)) p(x, y) \quad \forall x, y \in X .
$$

Then there exists a function $\beta: X \rightarrow[0,1)$ such that for each $x \in X$,

$$
\begin{gathered}
\beta(T x) \leq \beta(x), \\
\alpha\left(p\left(T^{n-1} x, T^{n} x\right)\right) \leq \beta(x) \quad \forall n \in \mathbb{N} .
\end{gathered}
$$

Here, we denote $T^{0}=I$ (the identity map).

Proof. Let $x \in X$ be given. From our hypothesis, we have

$$
\begin{aligned}
p\left(T^{n} x, T^{n+1} x\right) & \leq \alpha\left(p\left(T^{n-1} x, T^{n} x\right)\right) p\left(T^{n-1} x, T^{n} x\right) \\
& <p\left(T^{n-1} x, T^{n} x\right)
\end{aligned}
$$

for each $n \in \mathbb{N}$. So the sequence $\left\{p\left(T^{n-1} x, T^{n} x\right)\right\}_{n \in \mathbb{N}}$ is strictly decreasing in $[0,+\infty)$. Since $\alpha$ is an $\mathscr{M} \mathscr{T}$-function, by (g) of Theorem 11, we obtain

$$
0 \leq \sup _{n \in \mathbb{N}} \alpha\left(p\left(T^{n-1} x, T^{n} x\right)\right)<1 .
$$

Since $x \in X$ is arbitrary, we can define a new function $\beta$ : $X \rightarrow[0,1)$ by

$$
\beta(x):=\sup _{n \in \mathbb{N}} \alpha\left(p\left(T^{n-1} x, T^{n} x\right)\right) \quad \text { for } x \in X .
$$

It is obvious that for each $x \in X$, we have

$$
\begin{gathered}
\beta(T x) \leq \beta(x), \\
\alpha\left(p\left(T^{n-1} x, T^{n} x\right)\right) \leq \beta(x) \quad \forall n \in \mathbb{N} .
\end{gathered}
$$

As an interesting application of Theorem 13, we prove the following new fixed point theorems for Banach type maps.

Theorem 19. Let $(X, d)$ be a complete metric space, $p$ a generalized pseudodistance on $X$ with $p(x, y)=0$ implies $x=y$ for all $x, y \in X$, and $T: X \rightarrow X$ a selfmap. Suppose that

(a) there exists an $\mathscr{M} \mathscr{T}$-function $\alpha:[0, \infty) \rightarrow[0,1)$ such that

$$
p(T x, T y) \leq \alpha(p(x, y)) p(x, y) \quad \forall x, y \in X
$$

(b) one of the conditions (H1), (H2), (H3), (H4), and (H5) as in Theorem 13 holds.

Then $T$ admits a unique fixed point in $X$. Moreover, for each $x \in X$, the sequence $\left\{T^{n} x\right\}_{n \in \mathbb{N}}$ converges to the unique fixed point of $T$.
Proof. Denote $T^{0}=I$ (the identity map). Applying Theorem 18, there exists a function $\beta: X \rightarrow[0,1)$ such that for each $x \in X$,

$$
\begin{gathered}
\beta(T x) \leq \beta(x), \\
\alpha\left(p\left(T^{n-1} x, T^{n} x\right)\right) \leq \beta(x) \quad \forall n \in \mathbb{N} .
\end{gathered}
$$

For each $x \in X$, by (46), we get

$$
\begin{gathered}
p(x, T x)-\alpha(p(x, T x)) p(x, T x) \\
\leq p(x, T x)-p\left(T x, T^{2} x\right) .
\end{gathered}
$$

By exploiting the inequalities (47) and (48), we obtain

$$
\begin{aligned}
p(x, T x) \leq & \frac{1}{1-\alpha(p(x, T x))} p(x, T x) \\
& -\frac{1}{1-\alpha(p(x, T x))} p\left(T x, T^{2} x\right) \\
\leq & \frac{1}{1-\beta(x)} p(x, T x) \\
& -\frac{1}{1-\beta(T x)} p\left(T x, T^{2} x\right) .
\end{aligned}
$$

Let $\varphi: \mathbb{R} \rightarrow(0,+\infty)$ and $f: X \rightarrow \mathbb{R}$ be defined by

$$
\begin{gathered}
\varphi(t)=1 \quad \text { for } t \in \mathbb{R}, \\
f(x)=\frac{1}{1-\beta(x)} p(x, T x) \quad \text { for } x \in X,
\end{gathered}
$$

respectively. Then $\varphi$ is a nondecreasing function, and $f$ is a bounded below function. Clearly, $f(x)<+\infty$ for all $x \in X$. By (49), we obtain

$$
p(x, T x) \leq \varphi(f(x))(f(x)-f(T x)) \quad \forall x \in X .
$$

Hence we prove that $T: X \rightarrow X$ is a $(p, \varphi, f)$-Caristi type selfmap on $X$. Applying Theorem 13 , we know $\mathscr{F}(T) \neq \emptyset$. We claim that $\mathscr{F}(T)$ is a singleton set. Let $u, v \in \mathscr{F}(T)$. Then $T u=$ $u$ and $T v=v$. From (46), we have

$$
p(u, v)=p(T u, T v) \leq \alpha(p(u, v)) p(u, v)
$$

or

$$
(1-\alpha(p(u, v))) p(u, v) \leq 0,
$$

which implies $p(u, v)=0$. By our hypothesis, we get $u=v$ and our claim is proved. By the uniqueness of fixed point of $T$ and applying Theorem 13 again, the sequence $\left\{T^{n} x\right\}_{n \in \mathbb{N}}$ converges to the unique fixed point of $T$ for any $x \in X$.

As a direct consequence of Theorem 19, we obtain the following result.

Corollary 20. Let $(X, d)$ be a complete metric space, $p$ be a generalized pseudodistance on $X$ with $p(x, y)=0$ implies $x=$ $y$ for all $x, y \in X$, and $T: X \rightarrow X$ a selfmap. Suppose that 
(a) there exists $\gamma \in[0,1)$ such that

$$
p(T x, T y) \leq \gamma p(x, y) \quad \forall x, y \in X
$$

(b) one of the conditions (H1), (H2), (H3), (H4), and (H5) as in Theorem 13 holds.

Then $T$ admits a unique fixed point in $X$. Moreover, for each $x \in X$, the sequence $\left\{T^{n} x\right\}_{n \in \mathbb{N}}$ converges to the unique fixed point of $T$.

Applying Theorem 19, we obtain a generalization of the celebrated Banach contraction principle.

Corollary 21. Let $(X, d)$ be a complete metric space and $T$ : $X \rightarrow X$ a selfmap. Suppose that there exists an $\mathscr{M} \mathscr{T}$-function $\alpha:[0, \infty) \rightarrow[0,1)$ such that

$$
d(T x, T y) \leq \alpha(d(x, y)) d(x, y) \quad \forall x, y \in X .
$$

Then $T$ admits a unique fixed point in $X$. Moreover, for each $x \in X$, the sequence $\left\{T^{n} x\right\}_{n \in \mathbb{N}}$ converges to the unique fixed point of $T$.

Proof. By (55), we know that $T$ is continuous on $X$. Hence the conclusion follows from Theorem 19 immediately.

Remark 22. Theorems 13 and 19, Corollaries 15-21 all generalize and improve the celebrated Banach contraction principle.

\section{Acknowledgment}

In this research, the author was supported by Grant no. NSC 102-2115-M-017-001 of the National Science Council of the Republic of China.

\section{References}

[1] J. Caristi, "Fixed point theorems for mappings satisfying inwardness conditions," Transactions of the American Mathematical Society, vol. 215, pp. 241-251, 1976.

[2] S. Banach, "Sur les opérations dans les ensembles abstraits et leurs applications aux équations intégrales," Fundamenta Mathematicae, vol. 3, pp. 133-181, 1922.

[3] W. Oettli and M. Théra, "Equivalents of Ekeland's principle," Bulletin of the Australian Mathematical Society, vol. 48, no. 3, pp. 385-392, 1993.

[4] D. H. Hyers, G. Isac, and T. M. Rassias, Topics in Nonlinear Analysis \& Applications, World Scientific, River Edge, NJ, USA, 1997.

[5] W. Takahashi, Nonlinear Functional Analysis, Fixed Point Theory and Its Applications, Yokohama Publishers, Yokohama, Japan, 2000.

[6] A. Göpfert, C. Tammer, and C. Zălinescu, "On the vectorial Ekeland's variational principle and minimal points in product spaces," Nonlinear Analysis: Theory, Methods \& Applications, vol. 39, no. 7, pp. 909-922, 2000.

[7] A. Granas and C. D. Horvath, "On the order-theoretic Cantor theorem," Taiwanese Journal of Mathematics, vol. 4, no. 2, pp. 203-213, 2000.
[8] M. A. Khamsi, "Remarks on Caristi's fixed point theorem," Nonlinear Analysis: Theory, Methods \& Applications, vol. 71, no. 1-2, pp. 227-231, 2009.

[9] Z. Li, "Remarks on Caristi's fixed point theorem and Kirk's problem," Nonlinear Analysis: Theory, Methods \& Applications, vol. 73, no. 12, pp. 3751-3755, 2010.

[10] O. Kada, T. Suzuki, and W. Takahashi, "Nonconvex minimization theorems and fixed point theorems in complete metric spaces," Mathematica Japonica, vol. 44, no. 2, pp. 381-391, 1996.

[11] T. Suzuki, "Generalized distance and existence theorems in complete metric spaces," Journal of Mathematical Analysis and Applications, vol. 253, no. 2, pp. 440-458, 2001.

[12] T. Suzuki, "Generalized Caristi's fixed point theorems by Bae and others," Journal of Mathematical Analysis and Applications, vol. 302, no. 2, pp. 502-508, 2005.

[13] L.-J. Lin and W.-S. Du, "Ekeland's variational principle, minimax theorems and existence of nonconvex equilibria in complete metric spaces," Journal of Mathematical Analysis and Applications, vol. 323, no. 1, pp. 360-370, 2006.

[14] L.-J. Lin and W.-S. Du, "Some equivalent formulations of the generalized Ekeland's variational principle and their applications," Nonlinear Analysis: Theory, Methods \& Applications, vol. 67, no. 1, pp. 187-199, 2007.

[15] L.-J. Lin and W.-S. Du, "On maximal element theorems, variants of Ekeland's variational principle and their applications," Nonlinear Analysis: Theory, Methods \& Applications, vol. 68, no. 5, pp. 1246-1262, 2008.

[16] L.-J. Lin and W.-S. Du, "Systems of equilibrium problems with applications to new variants of Ekeland's variational principle, fixed point theorems and parametric optimization problems," Journal of Global Optimization, vol. 40, no. 4, pp. 663-677, 2008.

[17] W.-S. Du, "On some nonlinear problems induced by an abstract maximal element principle," Journal of Mathematical Analysis and Applications, vol. 347, no. 2, pp. 391-399, 2008.

[18] W.-S. Du, "Critical point theorems for nonlinear dynamical systems and their applications," Fixed Point Theory and Applications, vol. 2010, Article ID 246382, 16 pages, 2010.

[19] W.-S. Du, “On Latif's fixed point theorems," Taiwanese Journal of Mathematics, vol. 15, no. 4, pp. 1477-1485, 2011.

[20] W.-S. Du, "New approachs to the existence of fixed points for Caristitype mappings without lower semicontinuity assumptions," submitted.

[21] S. Al-Homidan, Q. H. Ansari, and J.-C. Yao, "Some generalizations of Ekeland-type variational principle with applications to equilibrium problems and fixed point theory," Nonlinear Analysis: Theory, Methods \& Applications, vol. 69, no. 1, pp. 126139, 2008.

[22] K. Włodarczyk and R. Plebaniak, "Maximality principle and general results of Ekeland and Caristi types without lower semicontinuity assumptions in cone uniform spaces with generalized pseudodistances," Fixed Point Theory and Applications, vol. 2010, Article ID 175453, 35 pages, 2010.

[23] S. Eshghinezhad and M. Fakhar, "Some generalizations of Ekeland's variational principle with applications to fixed point theory," Mathematical and Computer Modelling, vol. 57, pp. 1250-1258, 2013.

[24] P. Q. Khanh and D. N. Quy, "A generalized distance and enhanced Ekeland's variational principle for vector functions," Nonlinear Analysis: Theory, Methods \& Applications, vol. 73, no. 7, pp. 2245-2259, 2010. 
[25] P. Q. Khanh and D. N. Quy, “On generalized Ekeland's variational principle and equivalent formulations for set-valued mappings," Journal of Global Optimization, vol. 49, no. 3, pp. 381-396, 2011.

[26] J. H. Qiu and F. He, “p-distances, $q$-distances and a generalized Ekeland's variational principle in uniform spaces," Acta Mathematica Sinica, vol. 28, no. 2, pp. 235-254, 2012.

[27] J. R. Jachymski, "Caristi's fixed point theorem and selections of set-valued contractions," Journal of Mathematical Analysis and Applications, vol. 227, no. 1, pp. 55-67, 1998.

[28] W. A. Kirk and L. M. Saliga, "The Brézis-Browder order principle and extensions of Caristi's theorem," Nonlinear Analysis: Theory, Methods \& Applications, vol. 47, no. 4, pp. 2765-2778, 2001.

[29] A. Petruşel and A. Sîntămărian, "Single-valued and multivalued Caristi type operators," Publicationes Mathematicae Debrecen, vol. 60, no. 1-2, pp. 167-177, 2002.

[30] Y. Feng and S. Liu, "Fixed point theorems for multi-valued contractive mappings and multi-valued Caristi type mappings," Journal of Mathematical Analysis and Applications, vol. 317, no. 1, pp. 103-112, 2006.

[31] W.-S. Du, "Some new results and generalizations in metric fixed point theory," Nonlinear Analysis: Theory, Methods \& Applications, vol. 73, no. 5, pp. 1439-1446, 2010.

[32] W.-S. Du, "On coincidence point and fixed point theorems for nonlinear multivalued maps," Topology and Its Applications, vol. 159, no. 1, pp. 49-56, 2012.

[33] W.-S. Du, "On approximate coincidence point properties and their applications to fixed point theory," Journal of Applied Mathematics, vol. 2012, Article ID 302830, 17 pages, 2012.

[34] W.-S. Du, "On generalized weakly directional contractions and approximate fixed point property with applications," Fixed Point Theory and Applications, vol. 2012, article 6, 22 pages, 2012.

[35] W.-S. Du, Z. He, and Y.-L. Chen, "New existence theorems for approximate coincidence point property and approximate fixed point property with applications to metric fixed point theory," Journal of Nonlinear and Convex Analysis, vol. 13, no. 3, pp. 459474, 2012.

[36] W.-S. Du, "New existence results and generalizations for coincidence points and fixed points without global completeness," Abstract and Applied Analysis, vol. 2013, Article ID 214230, 12 pages, 2013. 


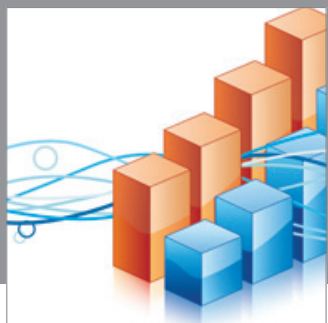

Advances in

Operations Research

mansans

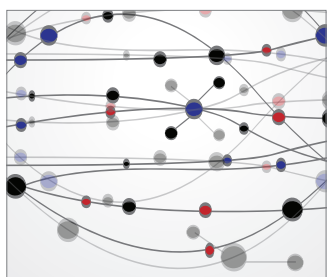

The Scientific World Journal
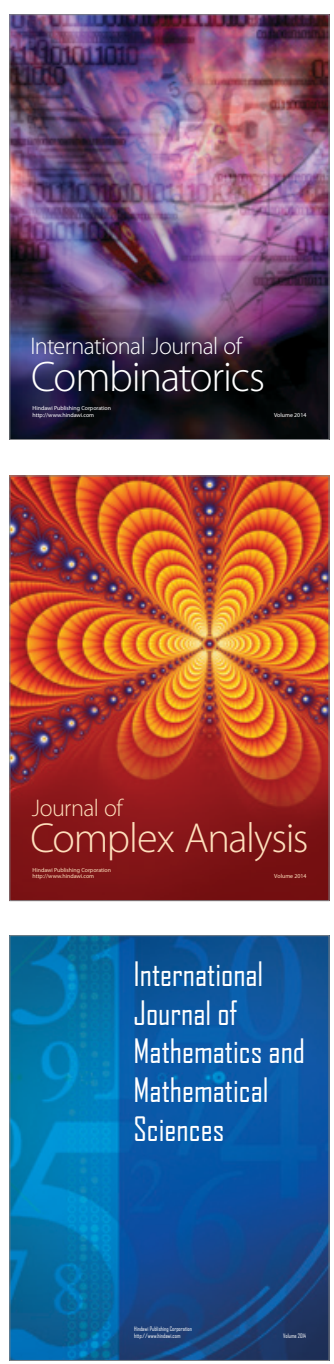
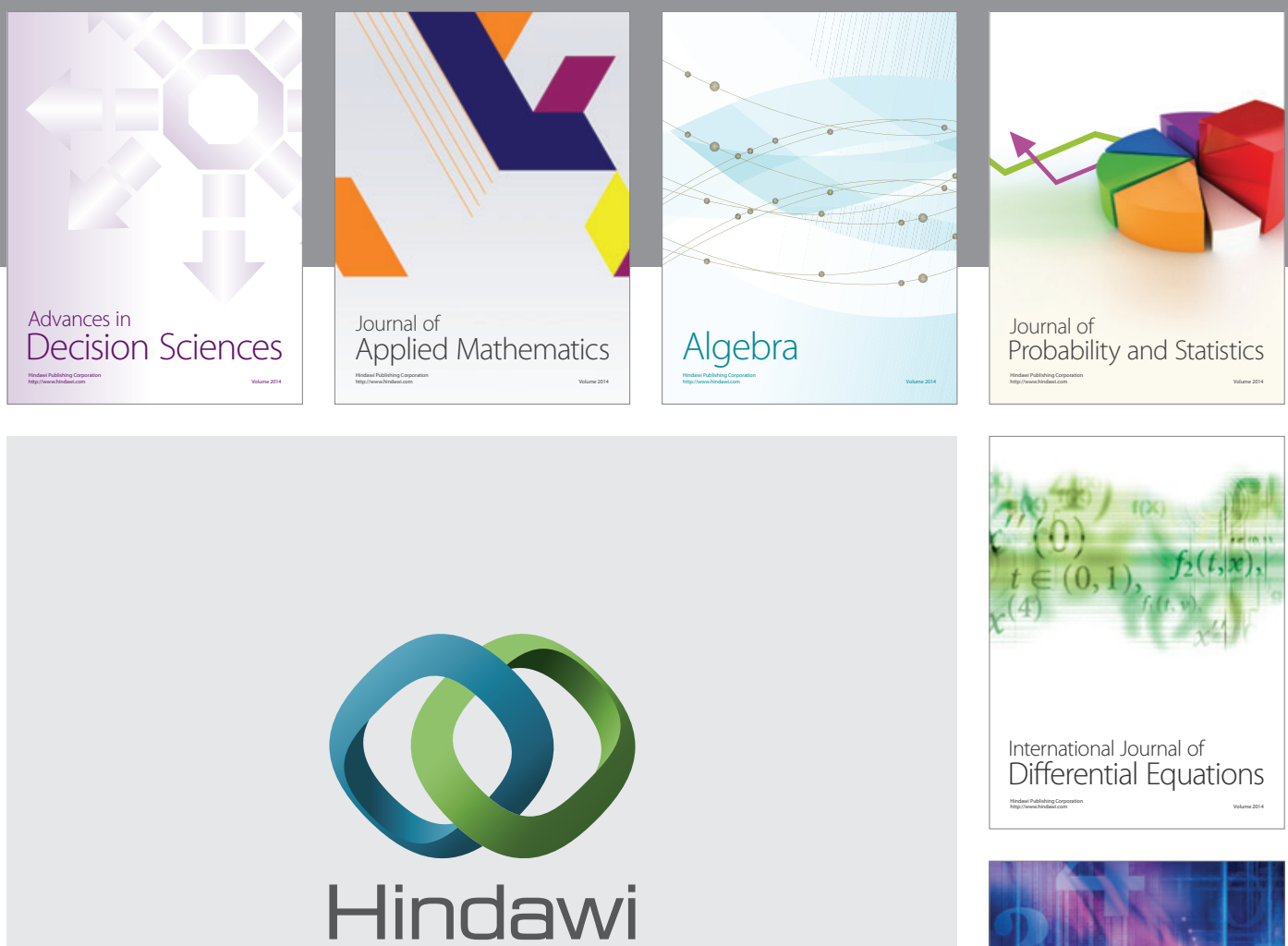

Submit your manuscripts at http://www.hindawi.com
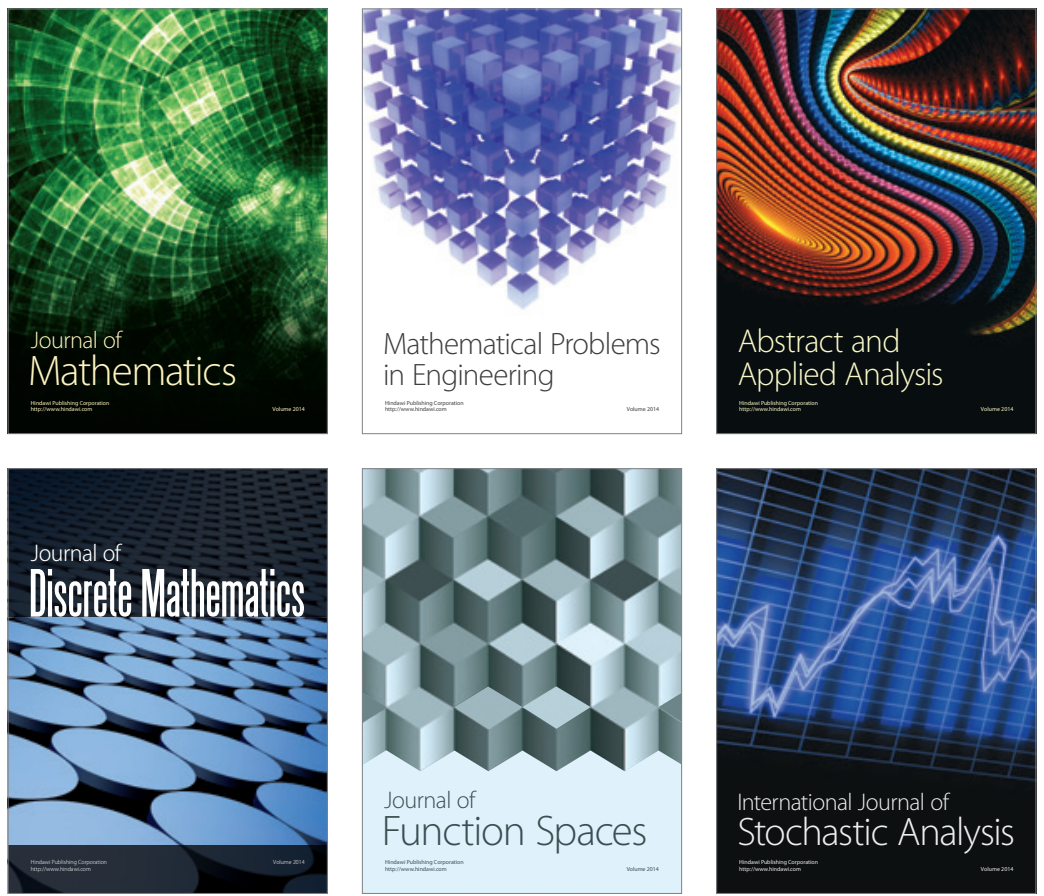

Journal of

Function Spaces

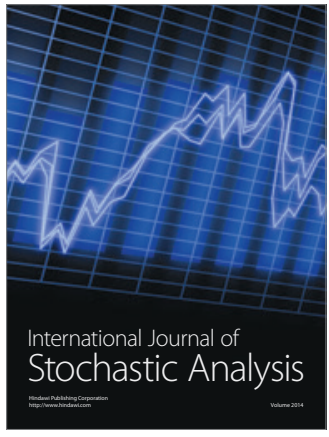

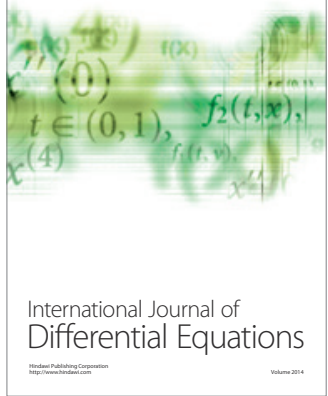
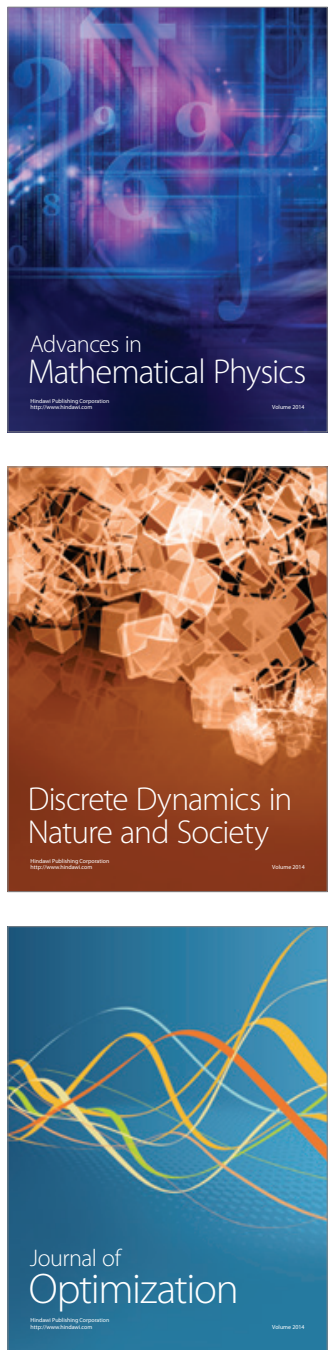